Computing and Informatics, Vol. 40, 2021, 195 215, doi: 10.31577/cai_2021_1_195

\title{
MORE EFFICIENT ON-THE-FLY VERIFICATION METHODS OF COLORED PETRI NETS
}

\author{
Cong He, Zhijun DinG* \\ Department of Computer Science and Technology \\ Tongji University \\ No. 4800, Caoan highway \\ Shanghai, China \\ e-mail: $\{1930766$, dingzj\}@tongji.edu.cn
}

\begin{abstract}
Colored Petri Nets (CP-nets or CPNs) are powerful modeling language for concurrent systems. As for CPNs' model checking, the mainstream method is unfolding that transforms a $\mathrm{CPN}$ into an equivalent $\mathrm{P} / \mathrm{T}$ net. However the equivalent $\mathrm{P} / \mathrm{T}$ net tends to be too enormous to be handled. As for checking $\mathrm{CPN}$ models without unfolding, we present three practical on-the-fly verification methods which are all focused on how to make state space generation more efficient. The first one is a basic one, based on a standard state space generation algorithm, but its efficiency is low. The second one is an overall improvement of the first. The third one sacrifices some applicability for higher efficiency. We implemented the three algorithms and validated great efficiency of latter two algorithms through experimental results.
\end{abstract}

Keywords: Model checking, CPN, on-the-fly, LTL, state space

Mathematics Subject Classification 2010: 93-A30

\section{INTRODUCTION}

CPNs are powerful graphical language for modeling concurrent systems introduced by Jensen in 1981 [9]. As a kind of high-level Petri nets, CPN is a Petri net that extends the type of place (token) to describe different data types. Moreover, arcs

* Corresponding author 
in CPN are labelled with arc expression functions to describe data operations; transitions in CPN are labelled with guard functions to describe branch conditions. In this way, CPN combines the capabilities of Petri nets and a high-level programming language. Success stories of $\mathrm{CPN}$ can be found in many industrial domains, such as network protocols [14, systematic softwares [11, 15, embedded systems [3, e-commerce systems [20], etc.

Explicit-state on-the-fly verification [4, 8, 7] is an universal optimization approach for model checking. It integrates state space generation, product automaton construction and counterexample detection (in LTL (Linear Temporal Logic) model checking, a counterexample is an accepting cycle in product automaton). An advantage of this approach is that the algorithm can give an answer without generating full state space. Though success stories of on-the-fly in $\mathrm{P} / \mathrm{T}$ nets clearly demonstrate its effectiveness and applicability, there are few works dedicated to directly applying on-the-fly in checking CPN models. As for checking CPN models, the mainstream approach is unfolding [16, 13, 12, 2], which transforms a CPN into an equivalent $\mathrm{P} / \mathrm{T}$ net and implements model checking on the latter. With unfolding, one can directly apply all successful optimization techniques which are difficult to extend to CPNs on the equivalent net, like Data Decision Diagram (DDD) [5, 1], $\mathrm{P}$-invariants [18. However, a big disadvantage of unfolding is that the equivalent $\mathrm{P} / \mathrm{T}$ nets transformed from a CPN tends to be too enormous to be handled, with much more places and transitions. Also, the transformed $\mathrm{P} / \mathrm{T}$ nets cannot directly describe the system to be verified. If a counterexample is detected by verification process, it is difficult to be directly reflected into the system, which is not friendly for debugging.

Concerned with checking CPN models without unfolding, we present a basic on-the-fly method, named full-info algorithm (FullInfo). It is based on the standard state space generation algorithm [10]. Its core idea is that once a new reachable state $m$ is generated, it calculates a set of all enabled binding elements (we call the set ENBE) in $m$ and stores ENBE together with marking. EnBE serves two purposes. One is to calculate successors of $m$. Another one is to help check atomic propositions carried by a Büchi automaton state during the generation of product automaton states (or product states for short). For example, some atomic propositions may check enabling of transitions, and enabling of transitions can be reflected by ENBE (for a transition $t$, if there exists an enabled binding element of $t$ in ENBE, $t$ is enabled. Otherwise, $t$ is not enabled). The algorithm is simple to implement but may generate much redundant information during on-the-fly. A great characteristic of on-the-fly is that it terminates exploration upon a counterexample is detected. Thus, in most cases, the set ENBE of every state $m$ is not fully utilized, cause many successors of $m$ have not been calculated before termination. Also, for some special LTL formulas whose atomic propositions are all related to numbers of tokens (this kind of LTL formulas are called LTLCardinality 1 formulas), ENBE can help

1 This terminology originated from MCC (Model Checking Contest) which is an annual competition for model checking. https://mcc.lip6.fr/. 
nothing, because checking these atomic propositions never refers to information in ENBE. This leads to waste of computing resources and low efficiency.

Besides Fullinfo, we introduce two more efficient state space generation methods integrated into on-the-fly, namely, minimum representative algorithm (MinRep) and dynamic exploration algorithm (DynExp). MinRep is inspired by canonical representative in [17]. Its core idea is that for every enabled transition $t$ in a newly calculated reachable state $m$, only a representative of enabled binding element of $t$ is initially calculated. While in DynExp, none enabled binding element is initially calculated in every newly generated reachable state $m$. Every enabled binding element in $m$ is calculated on demand when a new successor of $m$ needs generating to start a new path. However, without complete enabled transitions, DynExp is hard to check atomic propositions related to enabling of transitions. Thus, it is limited to LTLCardinality formulas.

In short, the main contributions of this paper are summarized as follows:

1. Concerned with LTL model checking of CPN without unfolding, we present an efficient on-the-fly verification method, named MinRep. It is an overall improvement of Fullinfo.

2. For LTLCardinality formulas, we present another more efficient on-the-fly verification method, named DynExp.

3. We implemented Fullinfo, MinRep and DynExp and did a number of experiments to demonstrate high efficiency of the latter two algorithms.

The rest of this paper is organized as follows: In Section 2, we introduce the definition of Colored Petri Nets and Linear Temporal Logics. In Section 3, we briefly introduce standard state space generation and on-the-fly verification. Then in Section 4. we specify a binding elements calculation problem from the core part of state space generation and on-the-fly. In Sections 5, 6, 7, we elaborate on Fullinfo, MinRep and DynExp. Their strengths and weaknesses are discussed as well. Implementation and experimental results are given in Section 8. Finally, in Section 9, we present our conclusion.

\section{PRELIMINARIES}

\subsection{Colored Petri Nets}

In this section, definitions of multi-set and non-hierarchical CPN are cited [10] and definitions of LTL are cited [6, 21]. As a matter of convenience, Bool $=\{$ false, true $\}$ is the set of Boolean types, where true and false are two predicates respectively. Type $[v]$ is the data type of variable $v$. Type $[e x]$ is the type of expression ex. EXPR is an expression constituted by elements from set $V$.

Definition 1 (Multi-set). Let $S=\left\{s_{1}, s_{2}, s_{3}, \ldots\right\}$ be a non-empty set. A multi-set $m$ is a function over $S: S \rightarrow \mathbb{N}$ that maps each element $s \in S$ into a non-negative 
integer $m(s) \in \mathbb{N}$ called the number of appearances (coefficient) of $s$ in $m$. A multiset $m$ can also be written as a sum (the operator ' ++ ' is a natural addition ' + ' when two elements $s_{1}, s_{2}$ are the same data type, otherwise ' ++ ' is just a junction symbol without real meaning):

$$
\sum_{s \in S} m(s)^{\prime} s=m\left(s_{1}\right)^{\prime} s_{1}++m\left(s_{2}\right)^{\prime} s_{2}++m\left(s_{3}\right)^{\prime} s_{3}++\ldots
$$

Operators: addition $(++)$, scalar multiplication $(* *)$, comparison $(\ll=)$, size $(|m|)$ and subtraction (--) are defined as follows:

- addition: $\forall s \in S,\left(m_{1}++m_{2}\right)(s)=m_{1}(s)+m_{2}(s)$,

- scalar multiplication: $\forall s \in S,(n * * m)(s)=n * m(s)$,

- comparison: $m_{1} \ll=m_{2} \Leftrightarrow \forall s \in S, m_{1}(s) \leq m_{2}(s)$,

- size: $|m|=\sum_{s \in S} m(s)$,

- when $m_{1} \ll=m_{2}$, subtraction is defined as: $\forall s \in S,\left(m_{2}--m_{1}\right)(s)=m_{2}(s)-$ $m_{1}(s)$.

Definition 2 (Non-hierarchical CPN). A non-hierarchical CPN is a nine-tuple $N=$ $(P, T, A, \Sigma, V, C, G, E, I)$, where $P, T, A$ are finite sets of places, transitions and arcs such that $P \cap T=\emptyset, A \subseteq P \times T \cup T \times P, \Sigma$ is finite set of non-empty color sets, $V$ is a finite set of typed variables such that Type $[v] \in \Sigma$ for all variables $v \in V, C: P \rightarrow \Sigma$ is a color set function that assigns a color set to each place, $G: T \rightarrow E X P R_{V}$ is a guard function that assigns a guard to each transition $t$ such that Type $[G(t)]=$ Bool, $E: A \rightarrow E X P R_{V}$ is an arc expression function that assigns an arc expression to each arc a such that Type $[E(a)]=C(p)_{M S}$ where $p$ is the place connected to the arc $a, I: P \rightarrow E X P R_{\emptyset}$ is an initialization function that assigns an initialization expression to each place $p$ such that Type $[I(p)]=C(p)_{M S}$. The variables of a transition $t$ are denoted $\operatorname{Var}(t), \operatorname{Var}(t) \subseteq V . \operatorname{Var}(t)$ includes all the variables appearing in $t$ 's guard $G(t)$ and arc expressions $E(a)$ for all $a \in A, a$ is connected to $t$.

Definition 3 (Enabling and firing rules). Let $N=(P, T, A, \Sigma, V, C, G, E, I)$ be a non-hierarchical CPN. A marking of $N$ is a function $M$ that maps each place $p \in P$ into a multi-set of tokens $M(p) \in C(p)_{M S}$. A binding of a transition $t$ is a function $b$ that maps each variable $v \in \operatorname{Var}(t)$ into a value $b(v) \in$ Type $[v]$. The set of all bindings for a transition $t$ is denoted $B(t)$, called $t$ 's binding space. A binding element is a pair $(t, b)$ such that $t \in T, b \in B(t)$. The set of all binding elements for a transition $t$ is denoted $B E(t)$, called $t$ 's binding element space. $B E(t)$ is defined by $B E(t)=\{(t, b) \mid b \in B(t)\}$. The set of all binding elements in a CPN is denoted $B E$, called binding element space. A binding element $(t, b) \in B E$ is enabled in a marking $M$ if and only if the following two properties are satisfied (denotation $G(t)\langle b\rangle$ expresses the evaluation of transition $t$ 's guard in the binding $b$ and it is either true or false; denotation $E(p, t)\langle b\rangle$ expresses the evaluation of arc $a$ 's arc expression and it is a multi-set): 
1. $G(t)\langle b\rangle=$ true;

2. $\forall p \in P, E(p, t)\langle b\rangle \ll=M(p)$.

When $(t, b)$ is enabled in $M$, it may occur and is leading to a marking $M^{\prime}$ (written $\left.M \stackrel{(t, b)}{\longrightarrow} M^{\prime}\right)$, such that $\forall p \in P, M^{\prime}(p)=(M(p)--E(p, t)\langle b\rangle)++E(t, p)\langle b\rangle$. A transition $t$ is enabled in a marking $M$ if and only if $\exists(t, b) \in B E(t),(t, b)$ is enabled in $M$.

Definition 4 (State space). For a marking $M$ and a marking $M^{\prime}$, if there exists an enabled binding element $(t, b)$ such that $M \stackrel{(t, b)}{\longrightarrow} M^{\prime}, M^{\prime}$ is said to be immediately reachable from $M$; if there exists an sequence of binding element $\left(t_{1}, b_{1}\right)\left(t_{2}, b_{2}\right) \ldots\left(t_{n}, b_{n}\right)$ such that $M \stackrel{\left(t_{1}, b_{1}\right)}{\longrightarrow} M_{1} \stackrel{\left(t_{2}, b_{2}\right)}{\longrightarrow} M_{2} \ldots \stackrel{\left(t_{n}, b_{n}\right)}{\longrightarrow} M^{\prime}, M^{\prime}$ is said to be reachable from $M$, written $M \stackrel{*}{\rightarrow} M^{\prime}$. The state space of a CPN consists of the set $R\left(m_{0}\right)=\left\{m \mid m_{0} \stackrel{*}{\rightarrow} m\right\}$ of states reachable from the initial state. Each state $m \in R\left(m_{0}\right)$ is called a reachable state ${ }^{2}$.

\subsection{Linear Temporal Logics}

Linear Temporal Logic (abbreviated as LTL) is used to describe properties of a system execution. It consists of a non-empty finite set of atomic propositions $A P$, Boolean operators $\neg$ (negation), $\vee$ (disjunction) and $\wedge$ (conjunction), and temporal operators $X$ (next), $U$ (until), $R$ (release), $F$ (eventually) and $G$ (always). In LTL model checking, the negation of a formula will be transformed into a Büchi automaton. There are many approaches to construct a Büchi automaton from the LTL formula [6, 19].

Definition 5 (Syntax of LTL). The syntax of LTL is defined as follows:

$$
\phi::=p|\neg \varphi| \varphi \vee \psi|\varphi \wedge \psi| X \varphi|\varphi U \psi| \varphi R \psi|F \varphi| G \varphi
$$

where $p$ is an atomic proposition and $\phi, \varphi, \psi$ are well-formed LTL formulas. Referring to MCC and Wolf's [21] provisions for atomic propositions, we make the following provisions for an atomic proposition $p$ :

$$
\begin{gathered}
p::=\text { TRUE } \mid \text { FALSE } \mid \text { FIREABLE }(t)(t \in T) \mid \text { DEADLOCK } \\
\mid k_{1} p_{1}+\cdots+k_{n} p_{n} \leq k\left(k_{i}, k \in \mathbb{Z}, p_{i} \in P\right)
\end{gathered}
$$

Let state $m$ be the current state, FIREABLE $(t)$ holds if only if $t$ is enabled in $m, D E A D L O C K$ holds if and only if there are no transitions are enabled in $m$, $k_{1} p_{1}+\cdots+k_{n} p_{n} \leq k$ holds if and only if $k_{1} M\left(p_{1}\right)+\cdots+k_{n} M\left(p_{n}\right) \leq k$ in $m$.

2 State is a snapshot of a system, marking is a distribution of tokens. Though a state $m$ can be uniquely identified by a marking $M$, they are different concepts. Throughout the paper, we use lower case $m$ (subscripts or superscripts will be used if necessary) to represent a state, upper case $M$ (subscripts or superscripts will be used if necessary) to represent the marking of $m$. 
Definition 6 (Semantics of LTL). Let $A P$ be a non-empty finite set of atomic propositions, $\xi=x_{0} x_{1} x_{2} \ldots$ be a sequence over alphabet $2^{A P}, \phi, \varphi, \psi$ be LTL formulas. We write $\xi_{i}$ for the suffix of $\xi$ starting at $x_{i}$. The semantics $\xi \models \phi(\xi$ models $\phi$ ) is defined as follows:

- $\xi \models p$, iff $p \in x_{0}$ for $p \in A P$,

- $\xi \models \neg \phi$, iff $\xi \not \models \phi$,

- $\xi \models \varphi \vee \psi$, iff $\xi \models \varphi$ or $\xi \models \psi$,

- $\xi \models X \phi$, iff $\xi_{1} \models \phi$,

- $\xi \models \varphi U \psi$, iff $\exists i \geq 0, \xi_{i} \models \psi \wedge\left(\forall j<i, \xi_{i}=\varphi\right)$.

Other operators $(\wedge, R, F, G)$ can be derived from the above operators $(X, U, \neg)$ : $\varphi \wedge \psi \equiv \neg(\neg \varphi \vee \neg \psi) ; \varphi R \psi \equiv \neg(\neg \varphi U \neg \psi) ; F \phi \equiv(T R U E) U \phi ; G \phi \equiv \neg(F \neg \phi)$.

\section{STANDARD STATE SPACE GENERATION AND ON-THE-FLY}

\subsection{Standard State Space Generation}

The standard state space generation [10] works on three sets: Node, UnProcessed, EDGES. Node stores reachable states. UnPROCESSED consists of states whose successors have not yet been calculated. EDGES stores arcs. As illustrated in Algorithm 1, the algorithm firstly initializes NoDE, UNPROCESSED with initial state $m_{0}$ and EDGEs with empty set. Then it selects a reachable state $m$ in UNPROCESSED and calculates all enabled binding elements in $m$. Each enabled binding element that occurs will lead to a reachable state $m^{\prime}$ and an arc from $m$ to $m^{\prime}$. If $m^{\prime}$ has not yet been encountered, it will be added into NoDE and UnProcessed. The algorithm terminates with full state space.

\subsection{On-the-Fly}

On-the-fly method was first proposed in [4. The main idea is integrating state space generation, product automaton construction and detecting counterexamples (in LTL model checking, a counterexample is an accepting cycle in product automaton). In more detail, for a given product state $p::(m, b)$ (a product state is composed by a reachable state $m$ and an automaton state $b$ ), it calculates a successor $m^{\prime}$ of $m$, and a successor $b^{\prime}$ of $b$. if all atomic propositions carried by $b^{\prime}$ are satisfied in $m^{\prime}$, then a product state $p^{\prime}::\left(m^{\prime}, b^{\prime}\right)$ is generated. If some conditions are triggered, on-thefly will implement counterexample detection, i.e., if on-the-fly finds the successor $p^{\prime}$ of $p$ is an encountered product state where it may form a cycle, then on-the-fly will check that. This idea can be illustrated by Algorithm 2. Line 1 is state space generation, lines 34 are the product state generation and the line 6 is counterexample detection. As for counterexample detection, there are several ways to do that. 


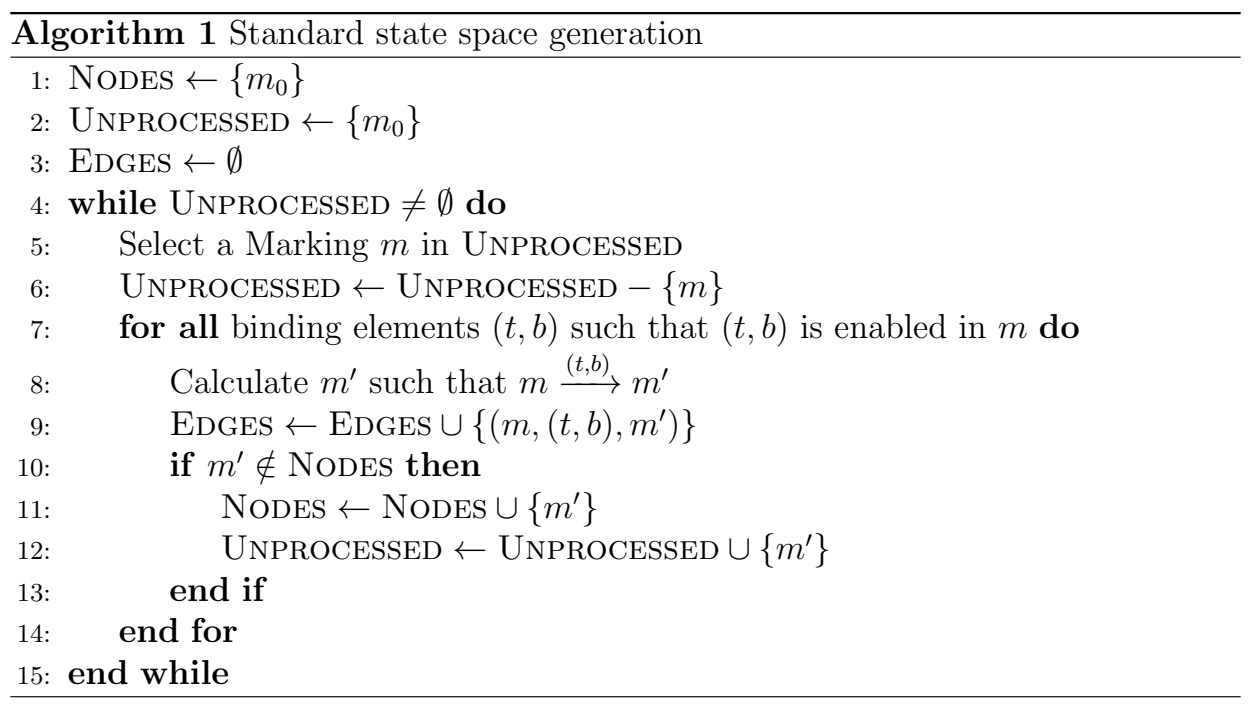

Like nested depth-first search algorithm [4], TCHECK $]^{3}$ algorithm [7] and DCHECK algorithm [7].

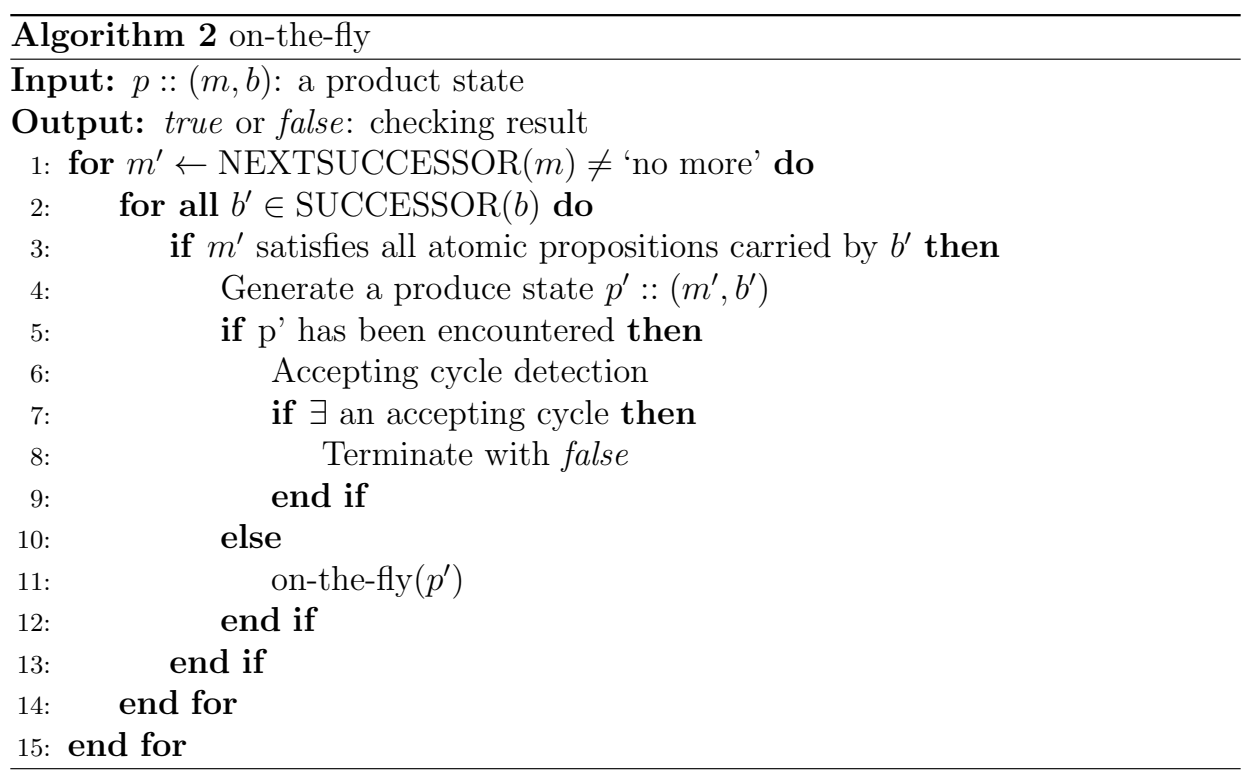

3 The main procedure of TCHECK and DCHECK are non-recursive functions, and they work much more efficiently than nested depth-first search. FullInfo, MinRep and DynExp are integrated into TCHECK algorithm. More details can be referred to [7]. 


\section{ENABLED BINDING ELEMENTS CALCULATION PROBLEM}

Enabled binding elements are vitaly important during state space generation. Firstly, all successors of a reachable state are controlled by enabled binding elements (lines 7 , 8 in Algorithm 1 and line 1 in Algorithm 2). Secondly, enabled binding elements plays a part in product state generation (line 3 in Algorithm 2), because some atomic propositions may check enabling of some transitions, i.e., FIREABLE $(t)$ atomic propositions. The core problem that we encounter is: given a reachable state $m$, in which way to explore binding element space $B E$ to find enabled binding elements in $m$ to calculate successors of $m$ and generate product states. Different solutions to this problem lead to huge different performances. Intuitively, we may come up with that upon a new reachable state $m$ is generated, explore $B E$ exhaustively at once to get all enabled binding elements ENBE in $m$ and store ENBE in case to use. In this way, every time on-the-fly backtracks to $m$, the process can easily fetch a next enabled binding element from ENBE to calculate another successor of $m$. This is exactly how Fullinfo works. We will detail it in the next section.

\section{FULLINFO}

The core idea of Fullinfo is very simple: upon getting a new reachable state $m$, it calculates a set of all enabled binding elements in $m$ called ENBE and stores ENBE together with marking $M$ immediately. Then it uses EnBE to generate different successors of $m$ and product states. The technical difficulties lie in how to get all enabled binding elements and how to manage them.

\subsection{How to Get All Enabled Binding Elements}

Traversing $t$ 's binding space $B(t)$ is essentially a combination problem that assigns a value $b(v) \in$ Type $[v]$ to each variable $v \in \operatorname{Var}(t)$. The binding space $B(t)$ can be depicted as a tree (we name it $t$ 's binding space tree). Assume that $t \in T$ is a transition, $|\operatorname{Var}(t)|=k$ such that $\operatorname{Var}(t)=\left\{v_{1}, v_{2}, \ldots, v_{k}\right\}$ and for each variable $v_{i}, \mid$ Type $\left[v_{i}\right] \mid=n_{i}$ such that Type $\left[v_{i}\right]=\left\{c_{i 1}, c_{i 2}, \ldots, c_{i n_{i}}\right\}$, then $B(t)$ can be depicted as a tree in Figure 1. The depth of this tree is equal to the number of variables in $\operatorname{Var}(t)$. All direct successors of a node are overall mapping cases of next variable. For example, the direct successors of node ' $v_{1}=c_{11}$ ' list complete mapping cases of next variable $v_{2}$, which is from $v_{2}=c_{21}$ to $v_{2}=c_{2 n_{2}}$ (we use horizontal ellipsis to represent all omitted nodes in its layer and vertical ellipsis to represent all omitted child nodes of one node). A path from root node to a leaf node is a specific binding of $t$, and all paths like this constitute $t$ 's binding space $B(t)$. We use a recursive function to traverse this tree to get all enabled binding elements. The function is presented in Algorithm 3. When the depth is lower than $|\operatorname{Var}(t)|$, the function tries to assign a color to the variable which corresponds to the depth and then recurves down. If the depth is equal to or greater than $|\operatorname{Var}(t)|$ which means the function 
reaches a leaf node and a complete binding $b$ is obtained, then it begins to check the enabling of $(t, b)$. The specific checking procedure lies in lines 2 2 . If $(t, b)$ is enabled, it will be added into ENBE. After implementing this function on each transition $t \in T$, the complete ENBE will be obtained.

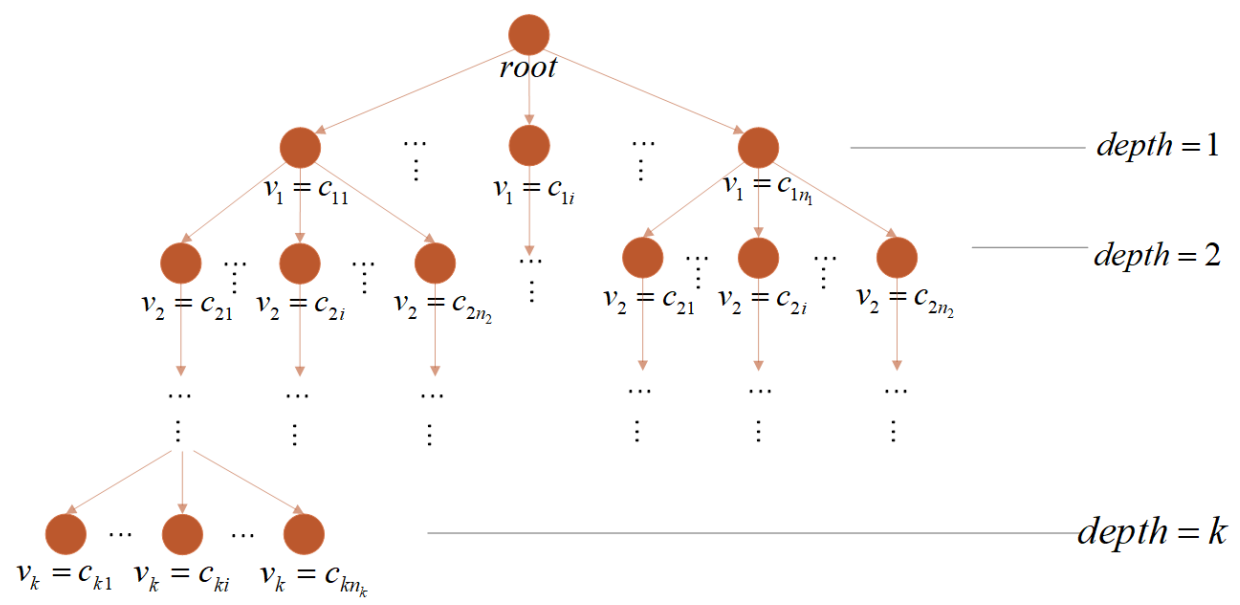

Figure 1. Binding space tree

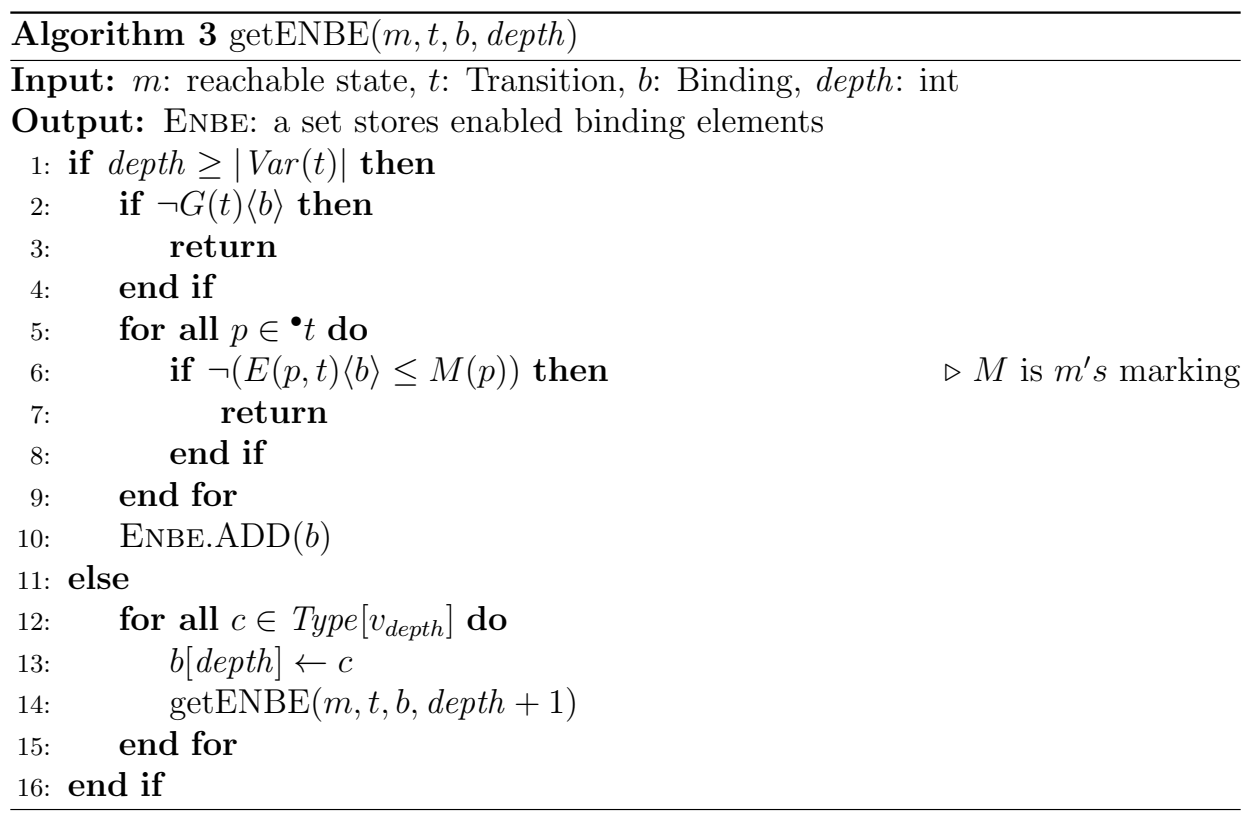




\subsection{How to Manage All Enabled Binding Elements}

In this subsection, we focus on how to take advantage of ENBE to serve for successor reachable states generation and product states generation. We use a two-level queue as data structure for ENBE. The first level queue stores enabled transitions, each enabled transition has a second level queue consisting of its bindings which render it enabled. Figure 2 is an example of

$$
\operatorname{ENBE}=\left\{\left(t_{1}, b_{11}\right),\left(t_{1}, b_{12}\right),\left(t_{2}, b_{21}\right),\left(t_{2}, b_{22}\right),\left(t_{2}, b_{23}\right),\left(t_{3}, b_{31}\right)\right\}
$$

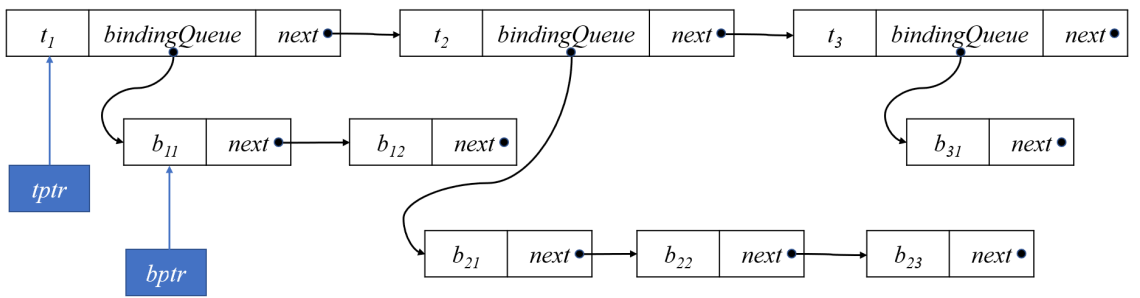

Figure 2. Data structure for ENBE

In the data structure of ENBE, there are two pointers, $t p t r$ and $b p t r$, respectively pointing to an enabled transition and a binding of it. They are used to represent an enabled binding element, i.e., in Figure 2 they represent $\left(t_{1}, b_{11}\right)$. Each time an enabled binding element occurs, bptr will move to next binding of the current queue. If next binding does not exist, i.e., it reaches the tail of the queue, tptr will move to next transition and bptr will point to the head of its bindingQueue. By this, the process can obtain different successors of a reachable state and this procedure is one possible way how line 7 in Algorithm 1 and line 1 in Algorithm 2 work.

Another crucial role of ENBE is to help generate a product state. For example, let $F \alpha$ be a LTL formula, where $\alpha$ is an atomic proposition FIREABLE $\left(t_{2}\right)$. During checking process, every state needs to check if $t_{2}$ is enabled in it. To do this, every state just needs to check its ENBE. If $t_{2}$ appears in the first level queue, it is enabled, otherwise it is not.

With Fullinfo, we can basically solve the enabled binding elements calculation problem. The two core parts, successor reachable states generation and product states generation, can be done easily with the aid of ENBE. But a conspicuous disadvantage is that it may generate much redundant information. Or in other words, many states' ENBE may not be fully utilized. For example, if on-the-fly reports a checking result without generating the whole state space, that means there must exist some states where some enabled binding elements have not yet occurred and these enabled binding elements remain to be redundant. Another case is when none of atomic propositions of the LTL formula is form of FIREABLE $(t)$ or DEADLOCK, ENBE can do nothing to help in the product state generation. This disadvantage is 
particularly obvious when a CPN's binding element space is huge or when on-thefly detects a counterexample along a path with few backtrackings. Here, Figure 3 is an example to demonstrate the second case. Figure 3 is a partial state space of a CPN. We use solid cycles to represent reachable states, arrows marked by transitions to represent enabled transitions in a reachable state, solid squares to represent enabled binding elements which have occurred and hollow squares to represent enabled binding elements which have not yet occurred. If on-the-fly detected a counterexample $S_{0} \rightarrow S_{1} \rightarrow S_{2} \rightarrow S_{0}$ after generating $S_{0}, S_{1}, S_{2}$ and then terminates, then the computing resources allocated for calculating the hollow squares are wasted because they had never been used during checking process. If on-the-fly went deeper along this path and detected a counterexample, the waste would be worse. Therefore, we need another algorithm to solve the enabled binding elements calculation problem.

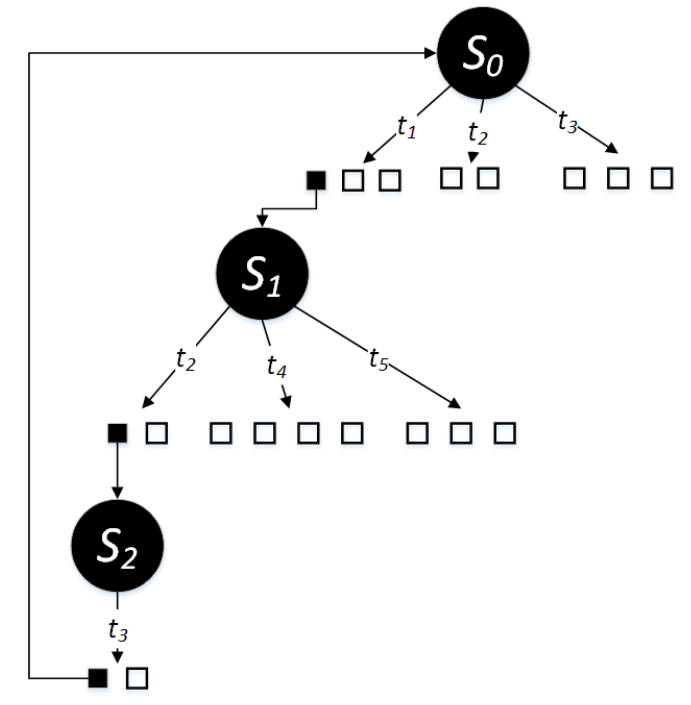

Figure 3. Partial state space

\section{MINREP}

In this section, we develop another solution to the enabled binding elements calculation problem. According to the definition of enabling of transitions (in Definition 3), if there exists one enabled binding element of a transition $t, t$ is proven to be enabled. Thus, as for checking atomic propositions during product state generation, it is unnecessary to calculate complete ENBE in each reachable state. The core idea of MinRep is to specify an order over $B(t)$ such that $(B(t), \prec)$ for each transition $t$. And for $t$ 's binding element space, this algorithm only initially calculates one en- 
abled representative which is the smallest enabled one in $B E(t)$. Certainly, if $t$ is not enabled, there will not be such a representative. This idea is inspired by canonical representative [17].

Before presenting the order $(B(t), \prec)$, we firstly specify an order $(C, \prec)$ over each color set $C \in \Sigma$. Here are the orders:

1. $(C, \prec): \forall c_{i}, c_{j} \in C, c_{i} \prec c_{j}$ iff $i<j$. Here the index $i, j$ can be arbitrarily defined. Typecially we use the index in data structure storing color set $C$, i.e., sequence table.

2. $(B(t), \prec): \operatorname{Var}(t)=\left\{v_{1}, v_{2}, \ldots, v_{n}\right\}, \forall b_{i}, b_{j} \in B(t), b_{i}=\left\langle c_{i 1}, c_{i 2}, \ldots, c_{i n}\right\rangle, b_{j}=$ $\left\langle c_{j 1}, c_{j 2}, \ldots, c_{j n}\right\rangle, b_{i} \prec b_{j}$ iff $\exists k, 1 \leq k \leq n, c_{i k} \prec c_{j k}$ and $\forall m, 1 \leq m<k, c_{i m}=$ $c_{j m} .(B(t), \prec)$ can be regarded as a lexicographical order induced by the vector of binding.

Calculating representative is similar to Algorithm 3 . The minor difference is that for MinRep, upon getting an enabled binding element, it terminates. More specifically, we just need to insert a terminate clause after line 10. All representatives are organized in a set, and we name it ENT. Here we use a queue to organize ENT. Figure 4 is an example of

$$
\mathrm{ENT}=\left\{\left(t_{1}, b_{11}\right),\left(t_{2}, b_{21}\right),\left(t_{3}, b_{31}\right)\right\}
$$

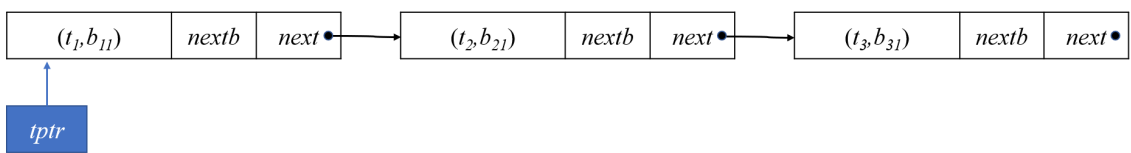

Figure 4. Data structure for ENT

In this data structure, tptr is a pointer pointing to a transition occurring last time and nextb is a binding that is prepared to calculate a successor reachable state next time (initially it equals to the binding part of the related representative). When on-the-fly backtracks to a reachable state $m$, the program uses nextb to calculate a successor $m^{\prime}$ of $\mathrm{m}$ and tries to check bindings behind (tptr $->$ nextb) to update nextb. If there are no more bindings related to tptr, tptr will move to next representative, which indicates the binding element space of prior transition has been explored exhaustively. The successor generation procedure is presented in Algorithm 4 where $(m . t p t r->t)$ is the transition of corresponding representative which pointed by tptr. Function UPDATE $(m)$ is to update $(t p t r->n e x t b)$. It regards the binding vector as a $n$-digit number, where $n$ equals to $|\operatorname{Var}(m \cdot t p t r->t)|$, and tries to implement increment ' ++ ' on this number. During the increment operation, it may trigger carries (lines 78 like numbers. Function NEXTSUCCESSOR $(m)$ is to get another successor of $m$ according to binding element (m.tptr $->t, m . t p t r->n e x t b)$. 
As for checking atomic propositions, ENT together with marking is right enough. As for FIREABLE $(t)$ propositions, MinRep checks ENT of current reachable state $m$. If there is a related representative of $t, t$ is enabled, otherwise, it is not. As for DEADLOCK propositions, MinRep also checks ENT of current reachable state $m$. If ENT is empty, the propositions are satisfied, otherwise, it is not. As for $k_{1} p_{1}+\cdots+k_{n} p_{n} \leq k$ propositions, MinRep checks them by the marking $M$ of current reachable $m$. if $k_{1} M\left(p_{1}\right)+\cdots+k_{n} M\left(p_{n}\right) \leq k$ holds, the propositions are satisfied, otherwise, it is not.

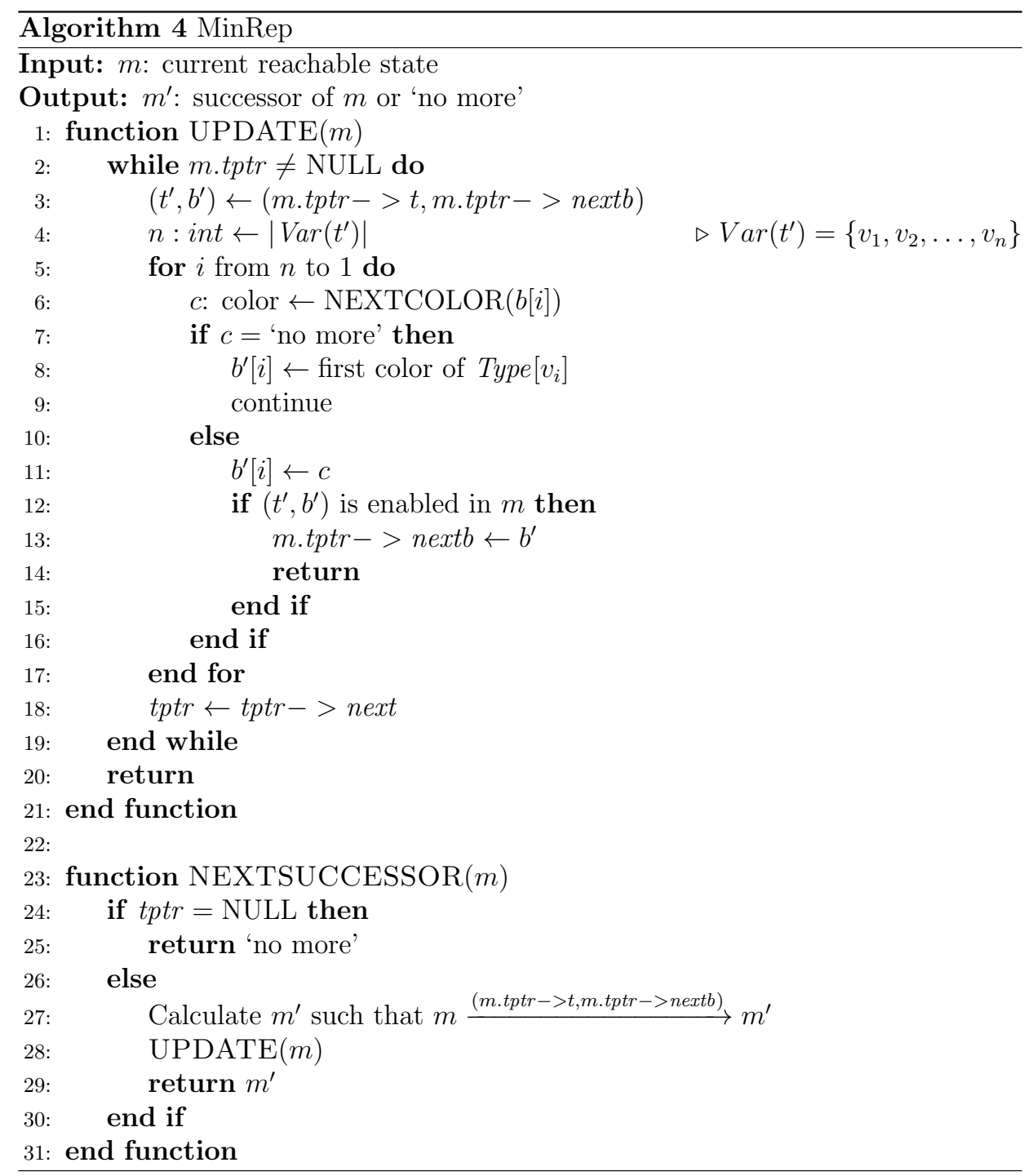


For each reachable state $m$, MinRep only calculates partial information from $m$ 's binding element space which is just enough to handle all kinds of atomic propositions. Compared with FullInfo, redundant information is much less and efficiency would be higher. But it has the same disadvantage that if all atomic propositions of the LTL formula are neither form of $D E A D L O C K$ nor FIREABLE $(t)$, i.e., LTLCardinality formulas, ENT can help nothing and remain to be redundant. So we need another more efficient algorithm to handle LTLCardinality formulas.

\section{DYNEXP}

In this section, we develop another algorithm dedicated to handling LTLCardinality formulas. As for checking atomic propositions of this formula type, it is unnecessary to calculate any enabled binding elements. The sole function of enabled binding elements here is to calculate successors. In order to be more efficient, DynExp will not initially calculate any enabled binding element in each newly calculated reachable state $m$. Instead of calculating all enabled binding elements at once, enabled binding elements are obtained dynamically. Once an enabled binding element is obtained, the algorithm will let it occur immediately and calculate a successor $m^{\prime}$ of $m$, then continue on-the-fly on $\mathrm{m}^{\prime}$. To obtain different successors of a given reachable state $m$ when on-the-fly backtracks to $m, D y n E x p$ extends the orders defined in Section 6 to the whole binding element space $B E$ such that $(B E, \prec)$, and each reachable state would record the binding element that occurred last time, called lastbe. In this way, when on-the-fly backtracks to $m$, it can check binding elements behind lastbe until an enabled one is detected or there are no more in $B E$.

Before presenting the order $(B E, \prec)$, we firstly specify an order $(T, \prec)$ over transition set $T$. They are defined as follows:

1. $(T, \prec): \forall t_{i}, t_{j} \in T, t_{i} \prec t_{j}$ iff $i<j$. Here the index value $i, j$ can be arbitrarily defined. Typically we use its index value in a specific data structure that stores the transition set.

2. $(B E, \prec): \forall\left(t_{i}, b_{i k}\right),\left(t_{j}, b_{j m}\right) \in B E,\left(t_{i}, b_{i k}\right) \prec\left(t_{j}, b_{j m}\right)$ iff $t_{i} \prec t_{j}$ or $i=j$, $b_{i k} \prec b_{j m}$.

With the aid of order $(B E, \prec)$ and lastbe, the algorithm can iterate over $B E$ exhaustively to get different enabled binding elements in each reachable state. We specify three functions to implement the idea, namely, NEXTBINDING $((t, b))$, $\operatorname{NEXTTRANSITION}((t, b))$ and $\operatorname{NEXTSUCCESSOR}(m)$. They are illustrated in Algorithm 5. Function NEXTBINDING $((t, b))$ is similar to function $\operatorname{UPDATE}(m)$ illustrated in Algorithm 4. It is to fetch $(t, b)$ 's next binding of transition $t$ according to order $(B E, \prec)$. Where function $\operatorname{NEXTCOLOR~}(c)$ is to get color $c$ 's next color in $c$ 's color set $C$ by order $(C, \prec)$. Function $\operatorname{NEXTTRANSITION}((t, b))$ is very simple. Its job is to fetch next transition of $t$ according to order $(T, \prec)$, and initiate the binding (lines 2325 ). If there are no more transitions, 'NULL' will be returned. 


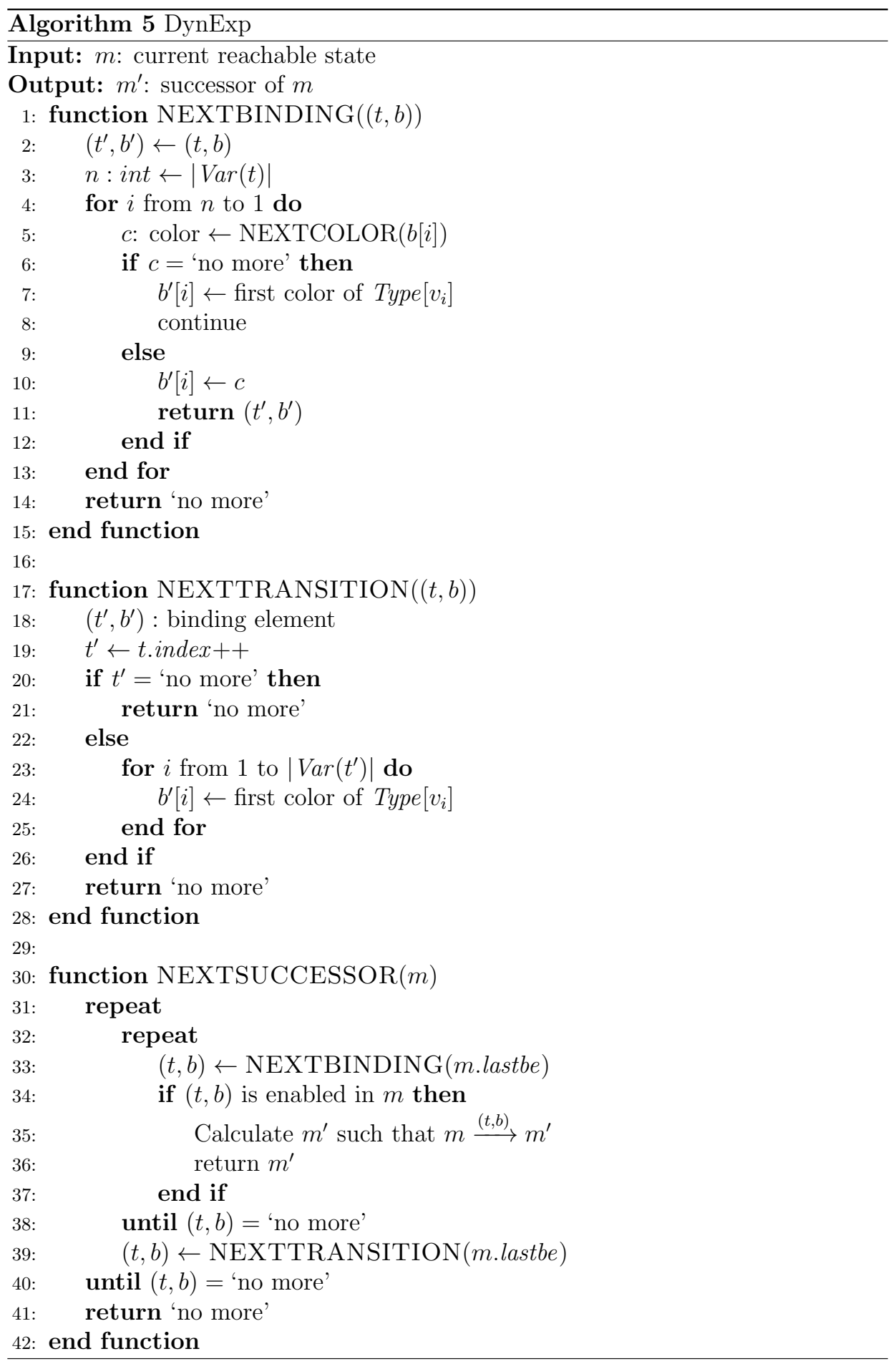


Function NEXTSUCCESSOR $(m)$ keeps calling NEXTBINDING(m.lastbe) to iterate over $B(t)$, trying to find an enabled one. If there are no more or do not exist at all, it will move to next transition by calling NEXTTRANSITION $(m$.lastbe). Upon obtaining an enabled binding element $(t, b),(t, b)$ will occur immediately leading to a successor $m^{\prime}$ of $m$ and terminates this function. Or if there are no more enabled binding element, NEXTSUCCESSOR $(m)$ will return 'no more'.

As for generating product states, we have nothing to worry about, because checking LTLCardinality formulas just need information of markings and markings are never absent.

Because upon getting a successor, the algorithm terminates exploring binding element space and continues on-the-fly, any enabled binding element and corresponding successor are calculated on demand during the checking process. Hence, no redundant information is generated and DynExp would be more efficient than Fullinfo and MinRep. However, it is limited to LTLCardinality formulas. It sacrifices applicability for greater efficiency.

\section{EXPERIMENT}

We implemented all three algorithms in $\mathrm{C}++$, and they are all integrated into the non-recursive on-the-fly TCHECK. The source code is available from:

- Fullinfo: https://github.com/Tj-Cong/EnPAC_CPN,

- MinRep: https://github.com/Tj-Cong/EnPAC_CPN_F,

- DynExp: https://github.com/Tj-Cong/EnPAC_CPN_C.

We get testing data from MCC. There are two kinds of models provided by MCC:

- Academic models: these were designed in universities by researcher, to benchmark some tools, to illustrate a typical situation or within the context of academic projects and cooperations.

- Industrial models: these where designed within the context of industrial projects.

Both kinds of models have practical meanings and each model is provided with a file describing it which can be found from https://mcc.lip6.fr/models.php. Each model can result in several instances due to the scaling parameter (the parameters are often indicated at the end of its instance name). There are two kinds of LTL formulas. One is called LTLCardinality formulas whose atomic propositions are all form of $k_{1} p_{1}+\cdots+k_{n} p_{n} \leq k$. Another kind is called LTLFireability formulas whose atomic propositions are all form of FIREABLE $(t)$. Based on this testing data, we have done two sets of experiments. One is designed to measure the performance on LTLcardinality formulas. Another one is designed to measure the performance on LTLfireability formulas. They are both implemented on a Linux PC with Intel(R) Core(TM) i7-7700HQ CPU @ 2.80 GHz and 16 GB RAM. Operating system is Ubuntu 18.04 LTS. 
As for the first experiment, all three algorithms were tested on four different instances with different size of binding element space. Each instance is checked by two formulas. Testing time for each formula is limited to 300 seconds, and if one algorithm does not finish checking one formula within 300 seconds, the corresponding table entry will be marked as "?". Memory for each formula is limited to 16 GB and if one algorithm cannot finish checking one formula within $16 \mathrm{~GB}$, the corresponding table entry will be marked as "Overflow". Of course, the three algorithms are set to traverse paths in the same order. The result is presented in Table 1. |States| represents the number of states explored by on-the-fly before termination. The unit of time is seconds; the unit of memory is MB; the size of binding element space $|B E|$ is calculated by:

$$
|B E|=\sum_{t \in T}\left(\prod_{v \in \operatorname{Var}(t)}(|\operatorname{Type}[v]|)\right) .
$$

From Table 1, we can find that DynExp always consumes the least time and memory. Thus, we can conclude that DynExp is the most efficient algorithm for LTLcardinality formulas, no matter with respect to memory consumption or time used. MinRep ranks the second and Fullinfo is the least efficient. Beginning from Formula 2 of DWM-COL-40, memory for Fullinfo overflows. And beginning from Formula 1 of GRA-COL-11, time for MinRep runs out of 300 seconds. When $|B E|$ goes larger, the advantage of dynamic exploration becomes more salient. By checking Formula 1 and Formula 2 of every instance, we can also find out that the more states on-the-fly explores, more obvious the advantage of DynExp is.

\begin{tabular}{|l|c|c|c|c|c|c|c|c|}
\hline Models & \multicolumn{2}{|c|}{ ALD-COL-10 } & \multicolumn{2}{c|}{ DWM-COL-40 } \\
\end{tabular}

${ }^{1}$ The full name is AirplaneLD-COL-0010.

${ }^{2}$ The full name is DatabaseWithMutex-COL-40.

${ }^{3}$ The full name is GlobalResAllocation-COL-11.

${ }^{4}$ The full name is DrinkVendingMachine-COL-16.

Table 1. Comparison on LTLCardinality formulas

As for the second experiment, FullInfo and MinRep were implemented on four different instances with different size of binding element space. Same as the first experiment, each instance is checked by two formulas and each formula is limited to 300 seconds and 16 GB. The result is presented in Table 2 Obviously, MinRep works more efficiently than FullInfo. Comparing Formula 2 of FR-COL-G005 with 
Formula 1 of GRA-COL-9, we can find that for MinRep, the memory consumption is much lower in GRA-COL-09, while for Fullinfo, the memory consumption is much higher in GRA-COL-9, because the size of binding element space is much bigger. We can also conclude that when $|B E|$ goes larger, the advantage of MinRep becomes more obvious. Also, the more states on-the-fly explores, more salient the advantage of MinRep is.

\begin{tabular}{|l|c|c|c|c|c|c|c|c|}
\hline Models & \multicolumn{2}{|c|}{ ALD-COL-50 } & \multicolumn{2}{c|}{ DWM-COL-40 } & \multicolumn{2}{c|}{ FR-COL-G005 $^{3}$} & \multicolumn{2}{c|}{ GRA-COL-9 $^{4}$} \\
\hline $\mid$ Places $\mid$ & \multicolumn{2}{|c|}{20} & \multicolumn{2}{c|}{11} & \multicolumn{2}{c|}{104} & \multicolumn{2}{c|}{5} \\
\hline Transitions & \multicolumn{2}{|c|}{15} & \multicolumn{2}{c|}{8} & \multicolumn{2}{c|}{136} & \multicolumn{2}{c|}{1003437} \\
\hline $\mid$ BE $\mid$ & \multicolumn{2}{|c|}{612} & \multicolumn{2}{c|}{12800} & 1 & 2 & 1 & 2 \\
\hline formulas & 1 & 2 & 1 & 2 & 115121 & 31812 & 36424 & $?$ \\
\hline States $\mid$ & 7 & 209 & 2077 & 9596 & 115 & $?$ \\
\hline Time (FullInfo) & 0.104 & 0.121 & 3.054 & 13.600 & 22.739 & 57.739 & $?$ & $? 300$ \\
\hline Time (MinRep) & 0.091 & 0.105 & 1.628 & 5.092 & 3.609 & 7.097 & 113.148 & $>300$ \\
\hline Memory (FullInfo) & 327.359 & 334.357 & 630.359 & 2222.359 & 1791.363 & 3894.363 & Overflow & Overflow \\
\hline Memory (MinRep) & 327.351 & 327.351 & 347.355 & 405.355 & 712.351 & 1553.348 & 400.348 & $?$ \\
\hline
\end{tabular}

${ }^{1}$ The full name is AirplaneLD-COL-0050.

${ }^{2}$ The full name is DatabaseWithMutex-COL- 40 .

${ }^{3}$ The full name is FamilyReunion-COL-L00200M0020C010P010G005.

${ }^{4}$ The full name is GlobalResAllocation-COL-09.

Table 2. Comparison on LTLFireability formulas

From the two experiments, we can conclude that no matter what kind of LTL formulas is, MinRep is always more efficient that FullInfo. While for LTLCardinality formulas, DynExp works best.

\section{CONCLUSIONS}

We have presented a basic state exploration method and two more efficient ones under the framework of on-the-fly. The basic one, Fullinfo, simply calculates all enabled binding elements for every newly generated reachable state. It is easy to implement but efficiency is low. MinRep is 'semi-dynamic'. It calculates all enabled transitions for every newly generated reachable state, but for each enabled transition, only a minimum representative of enabled binding elements is initially calculated, others are calculated dynamically on demand. While DynExp is 'fully-dynamic'. Every enabled binding element is calculated on demand by on-the-fly. As for applicability, FullInfo=MinRep $>$ DynExp. As for efficiency, DynExp $>$ MinRep $>$ FullInfo.

\section{Acknowledgement}

This work is partially supported by the National Key Research and Development Program of China under the Grant No. 2018YFB2100800 and the National Natural Science Foundation of China under Grant No. 61672381, and in part by the Fundamental Research Funds for the Central Universities under Grant No. 22120180508. 


\section{REFERENCES}

[1] Amparore, E. G.-Donatelli, S.-Beccuti, M.-Garbi, G.-Miner, A. S.: Decision Diagrams for Petri Nets: A Comparison of Variable Ordering Algorithms. In: Koutny, M., Kristensen, L., Penczek, W. (Eds.): Transactions on Petri Nets and Other Models of Concurrency XIII. Springer, Berlin, Heidelberg, Lecture Notes in Computer Science, Vol. 11090, 2018, pp. 73-92, doi: 10.1007/978-3-662-58381-4_4.

[2] Bergenthum, R.-Lorenz, R.-Mauser, S.: Faster Unfolding of General Petri Nets Based on Token Flows. In: van Hee, K. M., Valk, R. (Eds.).: Applications and Theory of Petri Nets (PETRI NETS 2008). Springer, Berlin, Heidelberg, Lecture Notes in Computer Science, Vol. 5062, 2008, pp. 13-32, doi: 10.1007/978-3-54068746-7_6.

[3] Christensen, S.-Jørgensen, J. B.: Analysing Bang \& Olufsen's Beolink@ Audio/Video System Using Coloured Petri Nets. In: Azéma, P., Balbo, G. (Eds.): Application and Theory of Petri Nets 1997 (ICATPN 1997). Springer, Berlin, Heidelberg, Lecture Notes in Computer Science, Vol. 1248, 1997, pp. 387-406, doi: 10.1007/3540-63139-9_47.

[4] Courcoubetis, C.-Vardi, M. Y.-Wolper, P.-Yannakakis, M.: Memory Efficient Algorithms for the Verification of Temporal Properties. In: Clarke, E. M., Kurshan, R.P. (Eds.): Computer-Aided Verification (CAV 1990). Springer, Berlin, Heidelberg, Lecture Notes in Computer Science, Vol. 531, 1991, pp. 233-242, doi: 10.1007/BFb0023737.

[5] Couvreur, J.-M.-Encrenaz, E.-Paviot-Adet, E.-Poitrenaud, D.Wacrenier, P.-A.: Data Decision Diagrams for Petri Net Analysis. In: Esparza, J., Lakos, C. (Eds.): Applications and Theory of Petri Nets 2002 (ICATPN 2002). Springer, Berlin, Heidelberg, Lecture Notes in Computer Science, Vol. 2360, 2002, pp. 101-120, doi: 10.1007/3-540-48068-4_8.

[6] Gastin, P.-Oddoux, D.: Fast LTL to Büchi Automata Translation. In: Berry, G., Comon, H., Finkel, A. (Eds.): Computer Aided Verification (CAV 2001). Springer, Berlin, Heidelberg, Lecture Notes in Computer Science, Vol. 2102, 2001, pp. 53-65, doi: 10.1007/3-540-44585-4_6.

[7] Geldenhuys, J.-Valmari, A.: More Efficient On-the-Fly LTL Verification with Tarjan's Algorithm. Theoretical Computer Science, Vol. 345, 2005, No. 1, pp. 60-82, doi: $10.1016 /$ j.tcs.2005.07.004

[8] Gerth, R.-Peled, D. A.-Vardi, M. Y.-Wolper, P.: Simple On-the-Fly Automatic Verification of Linear Temporal Logic. In: Dembinski, P., Sredniawa, M. (Eds.): Protocol Specification, Testing and Verification XV (PSTV 1995). Springer, Boston, MA, IFIP Advances in Information and Communication Technology, 1996, pp. 3-18, doi: $10.1007 / 978-0-387-34892-6 \_1$.

[9] Jensen, K.: Coloured Petri Nets and the Invariant-Method. Theoretical Computer Science, Vol. 14, 1981, No. 3, pp. 317-336, doi: 10.1016/0304-3975(81)90049-9.

[10] Jensen, K.-Kristensen, L. M.: Coloured Petri Nets: Modelling and Validation of Concurrent Systems. Springer, Berlin, Heidelberg, 2009, doi: 10.1007/b95112.

[11] Jørgensen, J. B.-Bossen, C.: Requirements Engineering for a Pervasive Health Care System. Proceedings of the $11^{\text {th }}$ IEEE International Conference on Requirements 
Engineering (RE 2003), Monterey Bay, CA, USA, September 2003, pp. 55-64, doi: 10.1109/ICRE.2003.1232737.

[12] Kordon, F.-Linard, A.-Paviot-Adet, E.: Optimized Colored Nets Unfolding. In: Najm, E., Pradat-Peyre, J.F., Donzeau-Gouge, V.V. (Eds.): Formal Techniques for Networked and Distributed Systems - FORTE 2006. Springer, Berlin, Heidelberg, Lecture Notes in Computer Science, Vol. 4229, 2006, pp. 339-355, doi: 10.1007/11888116_25.

[13] Kozura, V. E.: Unfoldings of Coloured Petri Nets. In: Bjørner, D., Broy, M., Zamulin, A. V. (Eds.): Perspectives of System Informatics (PSI 2001). Springer, Berlin, Heidelberg, Lecture Notes in Computer Science, Vol. 2244, 2001, pp. 268-278, doi: 10.1007/3-540-45575-2_27.

[14] Kristensen, L. M.-Jensen, K.: Specification and Validation of an Edge Router Discovery Protocol for Mobile Ad Hoc Networks. In: Ehrig, H., Damm, W., Desel, J., Große-Rhode, M., Reif, W., Schnieder, E., Westkämper, E. (Eds.): Integration of Software Specification Techniques for Applications in Engineering, Priority Program SoftSpez of the German Research Foundation. Springer, Berlin, Heidelberg, Lecture Notes in Computer Science, Vol. 3147, 2004, pp. 248-269, doi: 10.1007/978-3-54027863-4_15.

[15] Kristensen, L. M.-Jørgensen, J. B.-Jensen, K.: Application of Coloured Petri Nets in System Development. In: Desel, J., Reisig, W., Rozenberg, G. (Eds.): Lectures on Concurrency and Petri Nets (ACPN 2003). Springer, Berlin, Heidelberg, Lecture Notes in Computer Science, Vol. 3098, 2004, pp. 626-685, doi: 10.1007/9783-540-27755-2_18.

[16] McMillan, K. L.: Using Unfoldings to Avoid the State Explosion Problem in the Verification of Asynchronous Circuits. In: von Bochmann, G., Probst, D. K. (Eds.): Computer Aided Verification (CAV 1992). Springer, Berlin, Heidelberg, Lecture Notes in Computer Science, Vol. 663, 1993, pp. 164-177, doi: 10.1007/3-540-56496-9_14.

[17] Schmidt, K.: Integrating Low Level Symmetries into Reachability Analysis. In: Graf, S., Schwartzbach, M. I. (Eds.): Tools and Algorithms for the Construction and Analysis of Systems (TACAS 2000). Springer, Berlin, Heidelberg, Lecture Notes in Computer Science, Vol. 1785, 2000, pp. 315-330, doi: 10.1007/3-540-46419-0_22.

[18] Schmidt, K.: Using Petri Net Invariants in State Space Construction. In: Garavel, H., Hatcliff, J. (Eds.): Tools and Algorithms for the Construction and Analysis of Systems (TACAS 2003). Springer, Berlin, Heidelberg, Lecture Notes in Computer Science, Vol. 2619, 2003, pp. 473-488, doi: 10.1007/3-540-36577-x_35

[19] Tian, C.-Song, J.-Duan, Z.-Duan, Z.: LtlNfBa: Making LTL Translation More Practical. In: Liu, S., Duan, Z. (Eds.): Structured Object-Oriented Formal Language and Method (SOFL + MSVL 2015). Springer, Cham, Lecture Notes in Computer Science, Vol. 9559, 2016, pp. 179-194, doi: 10.1007/978-3-319-31220-0_13

[20] Wang, Z.-Luan, W.-Du, Y.-QI, L.: Composition and Application of Extended Colored Logic Petri Nets to E-Commerce Systems. IEEE Access, Vol. 8, 2020, pp. 36386-36397, doi: 10.1109/access.2020.2974883.

[21] Wolf, K.: How Petri Net Theory Serves Petri Net Model Checking: A Survey. In: Koutny, M., Pomello, L., Kristensen, L. (Eds.): Transactions on Petri Nets and 
Other Models of Concurrency XIV. Springer, Berlin, Heidelberg, Lecture Notes in Computer Science, Vol. 11790, 2019, pp. 36-63, doi: 10.1007/978-3-662-60651-3_2.
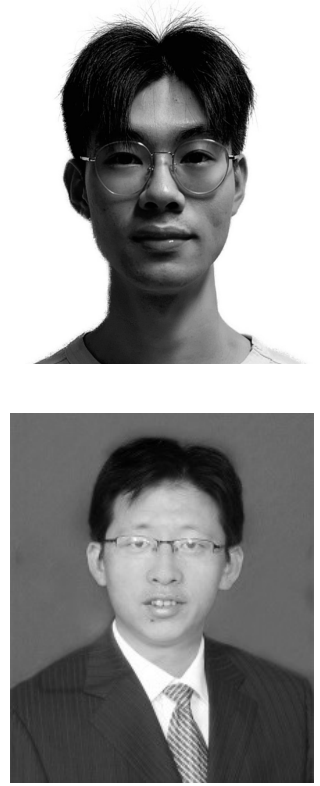

Cong He received his B.Sc. degree in computing science and technology from the Tongji University, Shanghai, China, in 2019. He is currently pursuing his M.Sc. degree in the Department of Computer Science and Technology, Tongji University, Shanghai, China. His current research interests include Petri nets and model checking.

Zhijun Ding received his M.Sc. degree from the Shandong University of Science and Technology, Tai'an, China, in 2001, and his Ph.D. degree from the Tongji University, Shanghai, China, in 2007. He is currently Professor with the Department of Computer Science and Technology, Tongji University. He has published over 100 papers in domestic and international academic journals and conference proceedings. His research interests are in formal engineering, Petri nets, services computing, and mobile internet. 